\title{
Exercise type and muscle fiber specific induction of caveolin-1 expression for insulin sensitivity of skeletal muscle
}

\author{
Yoon Sin $\mathrm{Oh}^{1}$, Hyo Jeong $\mathrm{Kim}^{2}$, \\ Sung Jin Ryu ${ }^{1}$, Kyung A Cho ${ }^{3}$, \\ Young Sik Park', Hyon Park, MiJung Kim ${ }^{5}$, \\ Chang Keun Kim ${ }^{2}$ and Sang Chul Park ${ }^{1,6}$ \\ ${ }^{1}$ Department of Biochemistry and Molecular Biology \\ The Aging and Apoptosis Research Center \\ Seoul National University College of Medicine \\ Seoul 110-799, Korea \\ ${ }^{2}$ Department of Health and Life Sciences \\ Korea National Sport University \\ Seoul 138-768, Korea \\ ${ }^{3}$ Department of Biochemistry and Molecular Biology \\ Chonnam University College of Medicine \\ Gwangju 501-746, Korea \\ ${ }^{4}$ Department of Sports Medicine \\ Kyung Hee University \\ Seoul 130-701, Korea \\ ${ }^{5}$ Department of Laboratory Medicine \\ University of Ulsan College of Medicine \\ Asan Institute for Life Sciences \\ Seoul 138-736, Korea \\ ${ }^{6}$ Corresponding author: Tel, 82-2-740-8244; \\ Fax, 82-2-744-4534; E-mail, scpark@snu.ac.kr
}

\section{Accepted 19 April 2007}

Abbreviations: EDL, extensor digitorum longus muscles; GLUT-4, glucose transporter-4; IR $\beta$, insulin receptor $\beta$; SD, Sprague Dawley; $\mathrm{SOL}$, soleus muscles

\begin{abstract}
It is well known that exercise can have beneficial effects on insulin resistance by activation of glucose transporter. Following up our previous report that caveolin-1 plays an important role in glucose uptake in L6 skeletal muscle cells, we examined whether exercise alters the expression of caveolin-1, and whether exercise-caused changes are muscle fiber and exercise type specific. Fifity week-old Sprague Dawley (SD) rats were trained to climb a ladder and treadmill for 8 weeks and their soleus muscles (SOL) and extensor digitorum longus muscles (EDL) were removed after the last bout of exercise and compared with those from non-exercised animals. We found that the expression of insulin related proteins and
\end{abstract}

caveolins did not change in SOL muscles after exercise. However, in EDL muscles, the expression of insulin receptor $\beta$ (IR $\beta$ ) and glucose transporter-4 (GLUT-4) as well as phosphorylation of AKT and AMPK increased with resistance exercise but not with aerobic exercise. Also, caveolin-1 and caveolin-3 increased along with insulin related proteins only in EDL muscles by resistance exercise. These results suggest that upregulation of caveolin- 1 in the skeletal muscle is fiber specific and exercise type specific, implicating the requirement of the specific mode of exercise to improve insulin sensitivity.

Keywords: caveolin 1; exercise therapy; glucose transporter type 4; muscle, skeletal; receptor, insulin

\section{Introduction}

Exercise increases glucose uptake, and improves glucose homeostasis as well as insulin sensitivity in the skeletal muscle. For its molecular mechanism, it is reported that exercise leads to the increased expression of glucose transporter (GLUT-4) as well as its translocation from cytosol to membrane in skeletal muscle (Zierath, 2002). The enhanced glucose uptake with exercise in skeletal muscle might be related to increased expression and activity of key proteins for insulin signaling such as insulin receptor (IR $\beta$ ), insulin receptor substrate (IRS)-1/2 and phosphatidylinositol 3-kinase (PI3-K) as well.

Caveolae are $50-100 \mathrm{~nm}$ sized invaginations in the cells (Sargiacomo et al., 1995). A wide variety of signaling proteins, recruited following activation of various receptors such as protein kinase C, G proteins, or receptor tyrosine kinase, are found to increase in caveolae (Schlegel et al., 1998). Caveolin is a 21-24 $\mathrm{kDa}$ protein in caveolae. The caveolin gene family consists of caveolins $-1,-2$, and -3 . Caveolin-1 and -2 are co-expressed in many cell types to form a hetero-oligomer in the plasma membrane (Schlegel and Lisanti, 2000), while the expression of caveolin-3 is muscle-specific (Song et al., 1996). Caveolin-1 has a scaffolding domain within its $\mathrm{NH} 2$-terminal region. Through this domain, caveolin-1 interacts with G-protein $\alpha$-subunits, $H$ Ras, Src-family tyrosine kinases, PKC isoforms, EGF-R, Neu, and eNOS and regulates their activities (Razani and Lisanti, 2001; Abulrob et al., 2004; Cao et al., 2004). 
Until now, the role of caveolin-1 in insulin signaling in muscle tissue has received little attention, probably caveolin-3 is expressed in a muscle specific manner and mutation of caveolin-3 cause limb girdle muscular dystrophy leading to apoptosis of skeletal muscle (Capanni et al., 2003; Smythe et al., 2003). However, it was not clear whether insulin resistance was induced by caveolin-3 deficiency or by muscle abnormality. Therefore, we have compared the roles of caveolin-1 and caveolin-3 in insulin signaling in differentiated L6 skeletal muscle cells (Oh et al., 2006).

We reported that caveolin-1 is involved in insulindependent glucose uptake via regulation of GLUT-4 translocation but not via IR $\beta$ pathway in muscle cells (Oh et al., 2006). Because both exercise- and insulin-stimuli increase glucose uptake through the translocation of GLUT-4, several studies have examined whether both these stimuli share the same signaling intermediaries or not (Hayashi et al., 1997).

Skeletal muscle is a mixture of two major fiber types (Schiaffino and Serrano, 2002) identified as type 1 (slow-twitch) and type 2 (fast-twitch) on the basis of their contractile and metabolic characteristics (Berchtold et al., 2000). The soleus muscle (type 1) is mitochondria-rich and mainly uses oxidative metabolism for energy production during aerobic exercises such as treadmill and swimming (Wang et al., 2004). In contrast, EDL muscle (type 2), which has low levels of mitochondrial content and oxidative enzymes, relies on glycolytic metabolism as a major energy source, for anaerobic exercise like ladder climbing (Berchtold et al., 2000; Olson and Williams, 2000).

Therefore, we asked two questions. One is, whether the exercise training would be related with modulation of caveolin status in the muscle tissues concomitantly with GLUT-4 adjustment and insulin sensitivity. Another one is, whether the exercise effect is muscle fiber specific or exercise type specific rather than general to all the muscles and exercise.

In this study, we provided resistance and aerobic exercise training to 50 week-aged rats for mobilizing the soleus and EDL muscles specifically, and examined whether the expression of caveolin-1 following each exercise was different and muscle type specific.

\section{Materials and Methods}

\section{Reagents}

The reagents used in this study and the sources from which they were purchased were as follows: Monoclonal anti-caveolin-1, -2 , and -3 antibodies (BD Transductions, Palo Alto, CA); polyclonal anti-
Akt, -AMPK, phospho-Akt and phosphor-AMPK antibodies (Cell Signaling Technology, Berverly, MA); antibodies against IR $\beta$ was from Upstate (Lake Placid, NY), anti-GLUT-4 antibody (Santa Cruz, CA); anti-tubulin antibody (Sigma, St. Louis, MO); HRP-conjugated anti-rabbit, anti-mouse and anti-goat antibodies (secondary antibodies) (Zymed, San Francisco, CA); and chemiluminescent detection systems (Pierce, Rockford, IL)

\section{Animals}

Female Sprague-Dawley rats, aged 50 weeks (weighing around $300 \mathrm{~g}$ ) were obtained from Dae Han Bio-Link, Korea and cared for according to the Guide Principles for the Care and Use of animals. They were, housed at $22 \pm 2{ }^{\circ} \mathrm{C}$ with $50 \%$ relative humidity maintained at a $12: 12 \mathrm{~h}$ dark-light cycle, and fed standard chow (Purina Mills Inc.) ad libitum.

After a week of adaptation, they were divided randomly into two groups: CON, (age-matched, non-exercised controls, $n=7$ ), RE, (resistance exercised with ladder climbing, $n=8$ ), and AE (aerobic exercised with treadmill, $n=8$ ).

\section{Exercise protocol}

A ladder made in our laboratory $(1 \mathrm{~m}$ of height, $2 \mathrm{~cm}$ grids and 85 degree of inclination) was used for the resistance training. After a week of adaptation, resistance training was begun using conical tubes attached to the base of tail with tape, a Velcro strap and a hook. Varying weights were inserted into the conical tube and the rats were forced to climb the ladder to the top by grooming action on the tail. The exercise was given at 2-3 p.m. three times per week. The first load started at $50 \%$ of their body weight and increased $180 \%$ of their body weight as the rats improved in their exercise ability. When the rats reached the top of the ladder, they were allowed to rest for $2 \mathrm{~min}$. Aerobic exercise was performed by treadmill running for 8 weeks, three times per week. During a week of adaptation, the rats were made to exercise on the treadmill at a velocity of $7 \mathrm{~m} / \mathrm{min}$ band, and during the main training, belt speed was $20 \mathrm{~m} / \mathrm{min}$ and duration of running time was increased from $40 \mathrm{~min}\left(1-2^{\text {th }}\right.$ week $)$ to $50 \mathrm{~min}\left(3-8^{\text {th }}\right.$ week).

\section{Western blot analysis}

Tissues were solubilized with a lysis buffer containing $1 \%$ SDS. Thirty $\mu \mathrm{g}$ of proteins from the lysates were resolved by $12 \%$ SDS-PAGE, transferred to nitrocellulose membranes, incubated with specific antibodies and visualized by blotting with HRP-conjugated secondary antibodies. 


\section{Immunohistochemistry}

Muscle tissue was cut in $4 \mu \mathrm{m}$ consecutive sections on a cryostat, and the sections were immediately collected on glass slides, to be used for hematoxylin-eosin ( $H \& E$ ) and immunohistological staining. Sections were preincubated in $3.0 \%$ $\mathrm{H}_{2} \mathrm{O}_{2}$ to quench endogenous peroxidase and then washed in TBS buffer $(0.05 \mathrm{M}, \mathrm{pH} 7.6)$. Slides were blocked in 5\% skim milk for $1 \mathrm{~h}$ at room temperature and then incubated at $4{ }^{\circ} \mathrm{C}$ overnight with caveolin-1 (1:100). The avidin-biotin peroxidase complex method following the LSAB kit was used, and slides were counterstained with hematoxylin. Slides were dehydrated sequentially in ethanol, cleared with xylenes, and mounted with Permount.

A

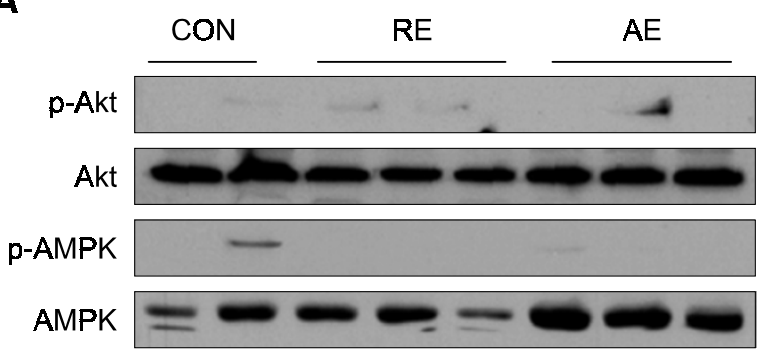

B

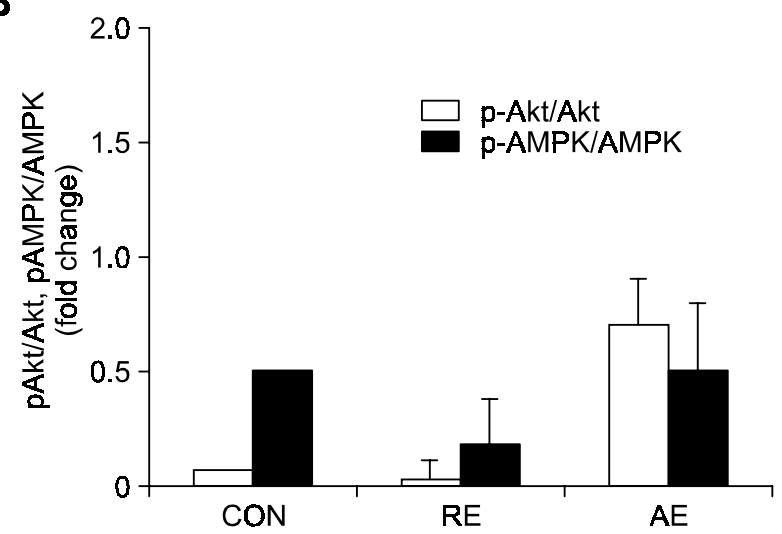

\section{Statistics}

All results are presented as the mean \pm S.E.M. Statistical analyses were performed using pair-wise comparisons ( $t$-test) and evaluated for Fisher's least significant differences. Statistical significance was assumed at $P<0.05$.

\section{Results}

We examined the effect of aerobic and resistance exercise on the expression of caveolins in the skeletal muscles in vivo, employing soleus and EDL muscle lysates obtained from the animals exercised as described above.

In order to test whether insulin sensitivity is fiber specific or exercise type specific, we analyzed phosphorylation of Akt and AMP-activated protein

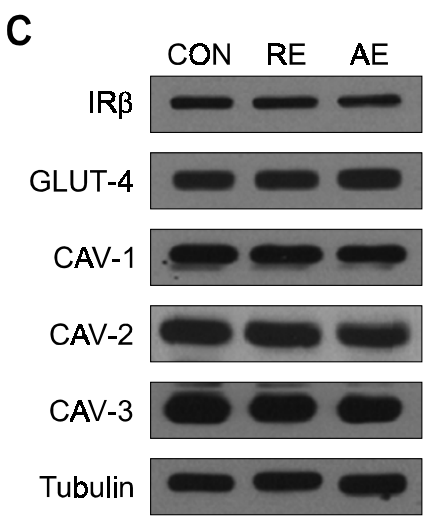

D

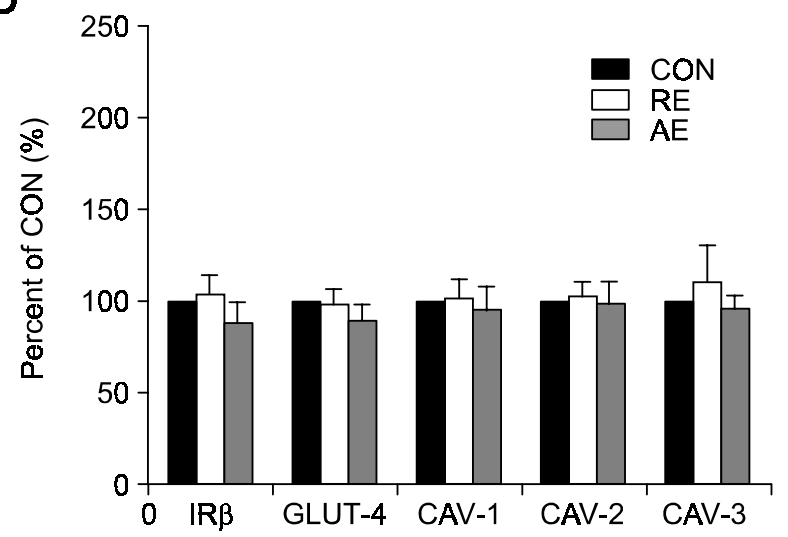

Figure 1. Expression of caveolins and insulin related proteins in soleus muscle during aerobic or resistance exercise. Soleus muscles obtained from exercised and non-exercised rats were studied as described in Materials and Methods. (A) Soleus muscle lysate $(30 \mu \mathrm{g})$ from resistance (RE), aerobic (AE) and non-exercised (CON) rat were subjected to SDS/PAGE (12\% gels) and immunoblotted with anti-Akt, AMPK, phospho-Akt (p-Akt) and phospho-AMPK (p-AMPK). (B) Quantitative analysis of Western blots. The relative fold changes in p-Akt and p-AMPK to its control bands on Western blots were quantitated by densitometric analysis. Each bar represents the mean $\pm \operatorname{SEM}(n=3)$. (C), Muscle tissue lysates were prepared as described in Figure $1 \mathrm{~A}$ and protein samples were analyzed by immunoblotting with anti-insulin receptor $\beta$ (IRß), GLUT-4, caveolin-1 (CAV-1), caveolin-2 (CAV-2), caveolin-3 (CAV-3) and tubulin (TUBULIN). (D) Quantitative analysis of Western blots. Relative abundance of each band was estimated by densitometric analysis. Each bar is the mean \pm S.E.M of fibers from 8 rats. 
A

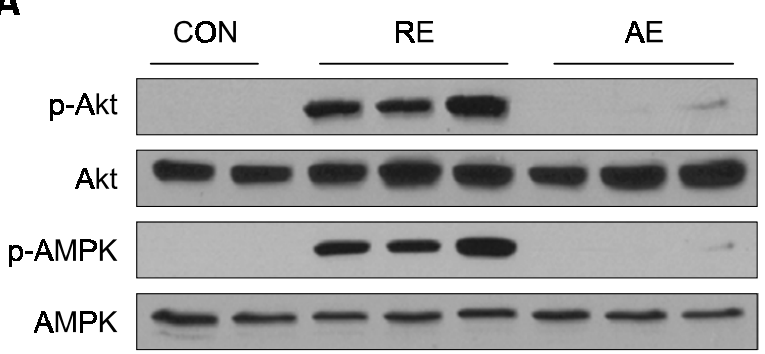

C

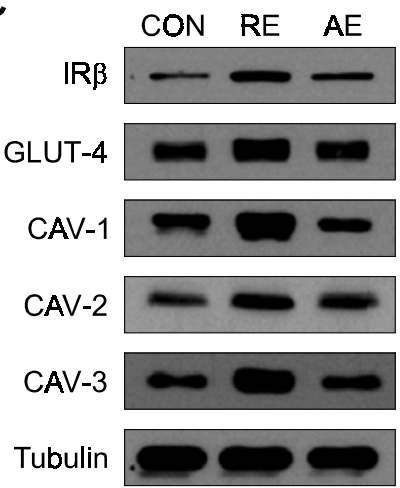

D

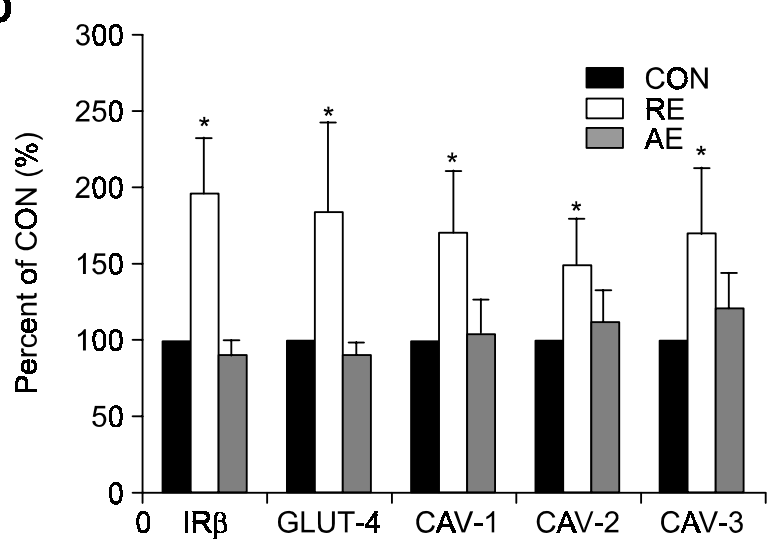

Figure 2. Expression of caveolins and insulin related proteins in EDL muscle during resistance exercise. (A) EDL muscles obtained from exercised and non-exercised rats were studied as described in Materials and Methods. EDL muscle lysate $(30 \mu \mathrm{g})$ from resistance (RE), aerobic (AE) and non-exercised (CON) rat were subjected to SDS/PAGE (12\% gels) and immunoblotted with anti-Akt, AMPK, phospho-Akt (p-Akt) and phospho-AMPK (p-AMPK). (B) Quantitative analysis of Western blots. The relative fold changes in p-Akt and p-AMPK to its control bands on Western blots were quantitated by densitometric analysis. Each bar represents the mean \pm SEM $(n=3)$. (C) Muscle tissue lysates were prepared as described in Figure $2 A$ and protein samples were analyzed by immunoblotting with anti-insulin receptor $\beta$ (IRß), GLUT-4, caveolin-1 (CAV-1), caveolin-2 (CAV-2), caveolin-3 (CAV-3) and tubulin (TUBULIN). (D) Quantitative analysis of Western blots. Relative abundance of each band was estimated by densitometric analysis. Each bar is the mean \pm S.E.M of fibers from 8 rats.

kinase (AMPK). Exercise specific increases in Akt activation (p-Akt) or AMPK (p-AMPK) by muscle fiber type were shown in Figure 1 ( $A$ and $B$ ) and Figure 2 ( $A$ and $B$ ). Phosphorylated -Akt and AMPK were high in only EDL muscle by resistance exercise (Figure 2A and B).

As shown in Figure 1, the expressions of the insulin related proteins, IR and GLUT-4, and caveolins did not change in the soleus muscle after either resistance or aerobic exercise training, compared to unexercised rats (Figure $1 C$ and $D$ ). In contrast, the expressions of insulin related proteins increased significantly after resistance exercise training in EDL muscles. Furthermore, caveolin-1, caveolin-2 and caveolin-3 also significantly increased $(P<0.05)$ (Figure 2C and D). Quantitative western blotting showed that expressions of caveolins increased 1.5-1.8 fold following the resistance exercise training parallel with the induction of insulin signaling molecules (Figure 2C). By immunohistochemistry, we confirmed that caveolin-1 in skeletal muscle fibers showed an increased staining of the cellular membrane of muscle fiber following resistance exercise in EDL muscle (Figure 3 ) as the same pattern of caveolin-1 protein level (Figure 2).

\section{Discussion}

Exercise increases whole body glucose utilization and improves insulin sensitivity (Zierath, 2002), both of which are mediated via increased expression of GLUT-4 as well as insulin-signaling molecules (Kirwan et al., 2000; Yu et al., 2001). The degree of 

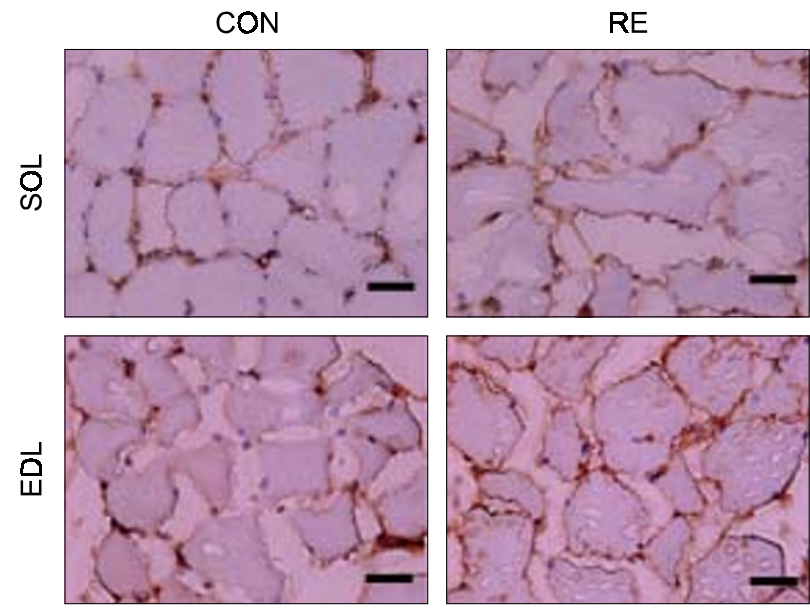

expression of the GLUT-4 as well as its translocation to membrane is suggested to be important for the regulation of glucose homeostasis (Dohm, 2002).

Previously, we found that caveolin-1 is involved in insulin-dependent glucose uptake via regulation of GLUT-4 translocation in muscle cells (Oh et al., 2006). Therefore, we questioned whether expression of caveolins would be also upregulated by exercise training with insulin sensitivity as the case of GLUT-4.

It was reported that insulin response to skeletal muscle is different between type 1 and type 2 muscles, as reported that peak insulin receptor (IR) tyrosine phosphorylation was reached after $6 \mathrm{~min}$ in soleus but 20 min in EDL (Song et al., 1999). Protein expression of the p85 subunit of PI3-K and Akt, but not of IR, IRS-1 was greater in oxidative muscle than glycolytic muscle.

In exercise responses, it is generally known that soleus muscle is involved in aerobic exercise, and EDL muscle is involved in resistance exercise (Trappe et al., 2004; Mittendorfer et al., 2005). However, some studies regarding the adaptation of insulin related proteins in the fiber type specific muscles to exercise training is confusing.

Rodnick et al. (1990) found an increase in GLUT-4 protein concentration in plantaris but not in soleus muscles of rats trained by voluntary wheel running. In another study, it was found that glucose uptake was increased in soleus muscles of rats trained by swimming; neither GLUT- 4 protein concentration nor mitochondrial enzyme levels were measured in the soleus (Ploug et al., 1990). Therefore, the mechanism of muscle protein balance by exercise has not been fully understood yet. Especially the fiber specific mode of exercise effect is not clear.

Under the assumption that expression of caveolins might influence the insulin sensitivity of muscle tissue, we monitored the exercise type

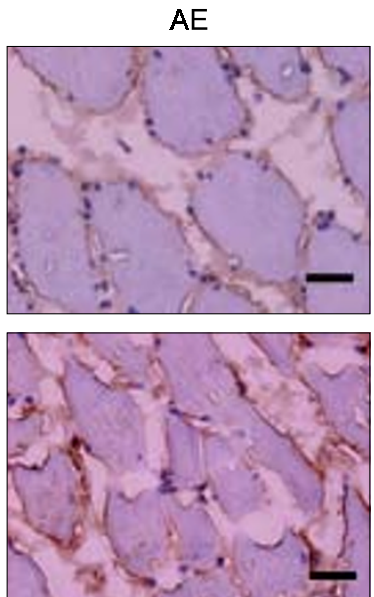

Figure 3. Caveolin-1 protein staining in SOL and EDL muscle in response to exercise. Soleus (SOL) and EDL (EDL) muscles obtained from exercised (RE, resistance exercise; $A E$, aerobic exercise) and non-exercised (CON) rats were studied as described in Materials and Methods. The caveolin-1 protein is predominantly localized at the cell membrane of the muscle fibers. dependent induction of caveolins in muscle fiber type specific manner. The metabolic response of muscle tissue to the exercise or insulin has been extensively studied (Atherton et al., 2004). However, the relation of insulin sensitivity with caveolin status in the muscle fiber specific and exercise type specific manner has not been studied yet.

The serine/threonine kinase AKT, a downstream target of PI3-kinase, has been implicated to play a role in glucose transport (Kohn et al., 1995). AMPactivated protein kinase (AMPK) also has been known as an important mediator of muscle contraction-induced glucose transport (Winder, 2001; Fujii et al., 2006) and is used to promote the glucose transport. We demonstrate here that the insulin sensitivity was not affected by aerobic exercise training in soleus muscle, but significantly increased only in EDL muscles by resistance exercise training (Figure 1 and 2). Accordingly, expression levels of IR $\beta$ as well as GLUT-4 significantly increased in EDL muscles only by resistance exercise, and not by aerobic exercise training (Figure 2). Also, we found that the expressions of caveolins increased in parallel with those of all the insulin sensitivity related proteins (Figure 2 and 3 ). This suggests that the expression of caveolins may be correlated with insulin sensitivity and be involved in the metabolic responses to exercise training in the muscle fiber-specific and exercise type-specific manners.

Although it is not clear whether changes in caveolins precede or follow those of GLUT-4 and IR $\beta$, our results demonstrate for the first time, that the expression of caveolin-1 is increased following an exercise bout in muscle fiber specific and exercise type specific pattern.

Caveolin-1 participates in cellular trafficking and that it contains many molecules including dynamin, and several SNARE proteins (Schnitzer et al., 1995; Liu et al., 2002). SNARE proteins and their acce- 
ssory proteins are required for the final fusion of GLUT-4-containing vesicles by integrating GLUT-4 protein into the plasma membrane (Saltiel, 2001). Therefore, induction of caveolin in the muscle tissue would facilitate the translocation of GLUT-4 to the membrane, which might contribute to the insulin sensitivity.

Caveolin-2 was reported to be co-expressed with caveolin-1 in general, and form hetero-oligomeric complexes (Rothberg et al., 1992; Kwak et al., 2005). As shown in Figure 1 and 2, patterns of caveolin-2 protein expressions are same as caveolin-1. Moreover, the expression of caveolin-3 increased in parallel with that of caveolin-1 in EDL muscles following exercise (Figure 2). It was reported that expression of caveolin-3 mRNA in the heart was higher in the exercise-trained SHR rats than in the non-exercise SHR rats (Lee et al., 2006). Also, we previously observed that caveolin-3 was co-expressed with caveolin-1 in skeletal muscle tissue and that they interact in L6 skeletal muscle cells (Capozza et al., 2005; Oh et al., 2006). Therefore, one might assume that increased expression of caveolin-1 would affect the expression of caveolin-3 (Figure 2).

The induction of caveolin-1 and -3 in EDL muscle with concomitant increase of $p-A K T$ and $p$-AMPK as well as induction of GLUT-4 only after resistance exercise training may suggest a specific mode of exercise to be applicable for the improvement of insulin sensitivity in type 2 muscle tissues.

Moreover, it was reported that caveolin-1 expression markedly decreased in aged rat skeletal muscle (Kawabe et al., 2001), and that the age-related reduction of insulin sensitivity is associated with age-related decrease in type 2 fiber muscle (Gaster et al., 2000). Therefore, it could be presumed that the restoration of type 2 fiber muscle by resistance exercise and the induction of caveolins in those muscle tissue would contribute greatly to improvement of insulin sensitivity of the skeletal tissue in aged organisms and consequently to prevention of age-related type 2 diabetes.

In conclusion, expression of insulin related proteins and caveolins were increased with insulin sensitivity by resistance exercise training in 50 week old rat skeletal muscle, especially of type 2 fibers, suggesting that up-regulation of caveolin-1 might be an important molecular response to exercise training. Studies on the elucidation of the underlying molecular mechanisms, currently underway in our laboratory, might lead to rational approaches to the treatment and management of insulin resistance and aging dependent type 2 diabetes.

\section{Acknowledgement}

This study was supported by the grants from Korea Science and Engineering Foundation (RII-2002-09705001-0 and RII-2002-097-00001-0), and Korea Research Foundation for Health Science and Ministry of Education (SNU BK 21 program).

\section{References}

Abulrob A, Giuseppin S, Andrade MF, McDermid A, Moreno $M$, Stanimirovic D. Interactions of EGFR and caveolin-1 in human glioblastoma cells: evidence that tyrosine phosphorylation regulates EGFR association with caveolae. Oncogene 2004;23:6967-79

Atherton PJ, Higginson JM, Singh J, Wackerhage $\mathrm{H}$. Concentrations of signal transduction proteins exercise and insulin responses in rat extensor digitorum longus and soleus muscles. Mol Cell Biochem 2004;261:111-6

Berchtold MW, Brinkmeier H, Muntener M. Calcium ion in skeletal muscle: its crucial role for muscle function, plasticity, and disease. Physiol Rev 2000;80:1215-65

Cao H, Sanguinetti AR, Mastick CC. Oxidative stress activates both Src-kinases and their negative regulator Csk and induces phosphorylation of two targeting proteins for Csk: caveolin-1 and paxillin. Exp Cell Res 2004;294:159-71

Capanni C, Sabatelli P, Mattioli E, Ognibene A, Columbaro M, Lattanzi G, Merlini L, Minetti C, Maraldi NM, Squarzoni $S$. Dysferlin in a hyperCKaemic patient with caveolin 3 mutation and in C2C12 cells after p38 MAP kinase inhibition. Exp Mol Med 2003;35:538-44

Capozza F, Cohen AW, Cheung MW, Sotgia F, Schubert W, Battista M, Lee H, Frank PG, Lisanti MP. Muscle-specific interaction of caveolin isoforms: differential complex formation between caveolins in fibroblastic vs. muscle cells. Am J Physiol Cell Physiol 2005;288:C677-91.

Dohm GL. Invited review: Regulation of skeletal muscle GLUT-4 expression by exercise. J Appl Physiol 2002;93: 782-7

Fujii N, Jessen N, Goodyear LJ. AMP-activated protein kinase and the regulation of glucose transport. Am J Physiol Endocrinol Metab 2006;291:E867-77

Gaster M, Poulsen P, Handberg A, Schroder HD, BeckNielsen H. Direct evidence of fiber type-dependent GLUT-4 expression in human skeletal muscle. Am J Physiol Endocrinol Metab 2000;278:E910-6

Hayashi T, Wojtaszewski JF, Goodyear LJ. Exercise regulation of glucose transport in skeletal muscle. Am J Physiol 1997;273:E1039-51

Kawabe JI, Grant BS, Yamamoto M, Schwencke C, Okumura $\mathrm{S}$, Ishikawa Y. Changes in caveolin subtype protein expression in aging rat organs. Mol Cell Endocrinol 2001;176:91-5

Kirwan JP, del Aguila LF, Hernandez JM, Williamson DL, O'Gorman DJ, Lewis R, Krishnan RK. Regular exercise enhances insulin activation of IRS-1-associated PI3-kinase in human skeletal muscle. J Appl Physiol 2000;88:797-803 
Kohn AD, Kovacina KS, Roth RA. Insulin stimulates the kinase activity of RAC-PK, a pleckstrin homology domain containing ser/thr kinase. Embo J 1995;14:4288-95

Kwak JO, Kim HW, Oh KJ, Kim DS, Han KO, Cha SH. Co-localization and interaction of organic anion transporter 1 with caveolin-2 in rat kidney. Exp Mol Med 2005;37:204-12

Lee YI, Cho JY, Kim MH, Kim KB, Lee DJ, Lee KS. Effects of exercise training on pathological cardiac hypertrophy related gene expression and apoptosis. Eur J Appl Physiol 2006;97:216-24

Liu P, Rudick M, Anderson RG. Multiple functions of caveolin-1. J Biol Chem 2002;277:41295-8

Mittendorfer B, Andersen JL, Plomgaard P, Saltin B, Babraj JA, Smith K, Rennie MJ. Protein synthesis rates in human muscles: neither anatomical location nor fibre-type composition are major determinants. J Physiol 2005;563:203-11

Oh YS, Cho KA, Ryu SJ, Khil LY, Jun HS, Yoon JW, Park SC. Regulation of insulin response in skeletal muscle cell by caveolin status. J Cell Biochem 2006;99:747-58

Olson EN, Williams RS. Remodeling muscles with calcineurin. Bioessays 2000;22:510-9

Ploug T, Stallknecht BM, Pedersen O, Kahn BB, Ohkuwa $\mathrm{T}$, Vinten J, Galbo H. Effect of endurance training on glucose transport capacity and glucose transporter expression in rat skeletal muscle. Am J Physiol 1990;259:E778-86

Razani B, Lisanti MP. Two distinct caveolin-1 domains mediate the functional interaction of caveolin-1 with protein kinase A. Am J Physiol Cell Physiol 2001;281:C1241-50

Rodnick KJ, Holloszy JO, Mondon CE, James DE. Effects of exercise training on insulin-regulatable glucose-transporter protein levels in rat skeletal muscle. Diabetes 1990; 39:1425-9

Rothberg KG, Heuser JE, Donzell WC, Ying YS, Glenney $\mathrm{JR}$, Anderson RG. Caveolin, a protein component of caveolae membrane coats. Cell 1992;68:673-82

Saltiel AR. New perspectives into the molecular pathogenesis and treatment of type 2 diabetes. Cell 2001;104: 517-29

Sargiacomo M, Scherer PE, Tang Z, Kubler E, Song KS, Sanders MC, Lisanti MP. Oligomeric structure of caveolin: implications for caveolae membrane organization. Proc Natl Acad Sci USA 1995;92:9407-11

Schiaffino S, Serrano A. Calcineurin signaling and neural control of skeletal muscle fiber type and size. Trends
Pharmacol Sci 2002;23:569-75

Schlegel A, Volonte D, Engelman JA, Galbiati F, Mehta P, Zhang XL, Scherer PE, Lisanti MP. Crowded little caves: structure and function of caveolae. Cell Signal 1998;10: 457-63

Schlegel A, Lisanti MP. A molecular dissection of caveolin-1 membrane attachment and oligomerization. Two separate regions of the caveolin-1 C-terminal domain mediate membrane binding and oligomer/oligomer interactions in vivo. J Biol Chem 2000;275:21605-17

Schnitzer JE, Liu J, Oh P. Endothelial caveolae have the molecular transport machinery for vesicle budding, docking, and fusion including VAMP, NSF, SNAP, annexins, and GTPases. J Biol Chem 1995;270:14399-404

Smythe GM, Eby JC, Disatnik MH, Rando TA. A caveolin-3 mutant that causes limb girdle muscular dystrophy type 1C disrupts Src localization and activity and induces apoptosis in skeletal myotubes. J Cell Sci 2003;116:4739-49

Song KS, Scherer PE, Tang Z, Okamoto T, Li S, Chafel M, Chu C, Kohtz DS, Lisanti MP. Expression of caveolin-3 in skeletal, cardiac, and smooth muscle cells. Caveolin-3 is a component of the sarcolemma and co-fractionates with dystrophin and dystrophin-associated glycoproteins. J Biol Chem 1996;271:15160-5

Song XM, Ryder JW, Kawano Y, Chibalin AV, Krook A, Zierath JR. Muscle fiber type specificity in insulin signal transduction. Am J Physiol 1999;277:R1690-6

Trappe TA, Raue U, Tesch PA. Human soleus muscle protein synthesis following resistance exercise. Acta Physiol Scand 2004;182:189-96

Wang YX, Zhang CL, Yu RT, Cho HK, Nelson MC, Bayuga-Ocampo CR, Ham J, Kang H, Evans RM. Regulation of muscle fiber type and running endurance by PPARdelta. PLoS Biol 2004;2:e294

Winder WW. Energy-sensing and signaling by AMPactivated protein kinase in skeletal muscle. J Appl Physiol 2001;91:1017-28

Yu M, Blomstrand E, Chibalin AV, Wallberg-Henriksson $\mathrm{H}$, Zierath JR, Krook A. Exercise-associated differences in an array of proteins involved in signal transduction and glucose transport. J Appl Physiol 2001;90:29-34

Zierath JR. Invited review: exercise training-induced changes in insulin signaling in skeletal muscle. J Appl Physiol 2002;93:773-81 